\title{
Hydrocarbon-Fueled Rocket Engine Plume Diagnostics: Analytical Developments \& Experimental Results
}

\author{
Gopal D. Tejwani, Gregory P. McVay, and Lester A. Langford \\ NASA Test Operations Group, John C. Stennis Space Center, MS 39529 \\ and \\ William W. St. Cyr \\ National Aeronautics and Space Administration \\ John C. Stennis Space Center, MS 39529
}




\section{Acknowledgments}

- RPTMBPO*

- John W. Bailey

- Randolph R. Holland

- Jorge F. Figuerora

- Lionel J. Dutreix

- Mark King

- Virgil K. Smith

- Jonathan A. Morris

- William M. Mitchell

- Brianne C. Guillot

- Jacob Christensen
- Craig A. Chandler

- Jared B. Grover

- Nathan E. Laborde

- Justin C. Junell

- Kerry D. Klein

- Robert L. Field

- Wanda Solano

- Daniel Allgood

- Satyajit Verma

- Ruby F. Menge

- Dane Freeman 


\section{Outline}

1. SSC Plume Diagnostics Background

2. Engine Health Monitoring Approach

3. Rocket Plume Spectroscopy Simulation Code

4. Spectral Simulation for 10 Atomic Species and for 11 Diatomic Molecular Electronic Bands

5. "Best" Lines for Plume Diagnostics for Hydrocarbon-Fueled Rocket Engines

6. Experimental Set Up for the Methane Thruster Test Program and Experimental Results

7. Summary and Recommendations 


\section{Background}

- Plume Diagnostics has Unique Capabilities for the Engine Health Monitoring

- Non-Intrusive

- Remote Sensing

- Extremely Sensitive

- Highly Specific in Many Cases

- Precursive

- Successful Application to the Space Shuttle Main Engine (SSME) Testing Program at SSC since 1989

- High-Temperature Emission in the Mach Diamond Regions

- Quantification of Atomic Species by using a Spectral Simulation Code

- Identification of Effluent Materials by the Engine Diagnostics Control (EDC)

- Particularly Useful in the Alternate Turbopump Development (ATD) for High Pressure Fuel Turbopump (HPFTP) and High Pressure Oxidizer Turbopump (HPOTP)

- Numerous Successful Identifications of SSME Alloys in the Plume by the EDC. Many Quantification Results Validated

- Presence of $\mathrm{CO}_{2}, \mathrm{C}, \mathrm{CH}, \mathrm{CO}, \mathrm{CN}$, and $\mathrm{NO}$ in addition to $\mathrm{OH}$ and $\mathrm{H}_{2} \mathrm{O}$ in the Hydrocarbon-Fueled Rocket Engines 


\section{Engine Health Monitoring Approach}

- Very Wide Spectral Range Coverage

- High Resolution: 300 to 430 nm (for Ni, Cr, Fe, Mn, Co, Ag, Cu, Al, $\mathrm{Ca}$, and Pd)

- Low Resolution: 300 to 820 nm (Oxides, Hydrides and Hydroxides)

- Identify Metallic Elemental Species in the Plume

- Quantify Elemental Plume Contaminants

- Identify and Quantify the Alloy Combination on the Basis of Elemental Concentrations

- Most Probable Hardware Sources for Effluent Material(s) 


\section{Spectral Simulation Code for Hydrocarbon-Fueled Engine Plumes}

- Rocket Plume Spectral Simulation Code (RPSSC) is Based on SSC Line-by-Line (LBL) Code which itself is Based on NASA Ames LBL Code

- Voigt Line Profile

- Convolution with an Entrance Slit Function

- Optically Thin or Optically Thick Environment

- Non-Uniform Wavelength Computation Interval

- Multiple Layer Scheme for Radiance Computations

- All Significant Atomic Transitions for Ten SSME Elements: $\mathrm{Ni}, \mathrm{Cr}, \mathrm{Fe}, \mathrm{Co}, \mathrm{Mn}, \mathrm{Ag}, \mathrm{Cu}, \mathrm{Pd}, \mathrm{Al}$, and $\mathrm{Ca}$

- Eleven Electronic Band Systems for Six Diatomic Molecules 


\section{Rocket Plume Spectral Simulation Code}

- Six Diatomic Species: $\mathrm{OH}, \mathrm{CH}, \mathrm{C}_{2}, \mathrm{CO}, \mathrm{CN}$, and $\mathrm{NO}$

- Eleven Electronic Band Systems (300 to $850 \mathrm{~nm}$ )

- OH 3064 A Band System

- $\mathrm{C}_{2}$ Swan Band System

- CH 3900 Å Band System

- CH 4300 Å Band System

- CN Violet Band System

- CO+ Comet-Tail Band System

- CN Red Band System

- $\mathrm{C}_{2}$ Phillips Band System

- CO Asundi Band System

- NO Beta Band System

- NO Gamma Band System 


\section{Atomic Emission Line Parameters}

1. Doppler Broadened Linewidth at Half-Height

2. Pressure Broadened Linewidth at Half-Height

3. Electronic Multiplicity of the Upper State

4. Electronic Term Energy of the Upper State

5. Einstein Transition Probability Coefficient

6. Atomic Line Center 
Table 1. Molecular and Spectroscopic Parameters for $\mathrm{C}_{2}$ Swan Band System

\begin{tabular}{|c|c|c|c|c|c|c|c|c|}
\hline & \multicolumn{4}{|c|}{$\begin{array}{l}\text { Nuclear Spin for Homonuclear } \\
\text { Molecules }\end{array}$} & Number of $E$ & tronic Levels & \multicolumn{2}{|c|}{$\begin{array}{c}\text { Reduced Mass in Atomic Weight } \\
\text { Units }\end{array}$} \\
\hline & \multicolumn{2}{|c|}{0.} & \multicolumn{2}{|c|}{0.0} & \multicolumn{2}{|c|}{10} & \multicolumn{2}{|c|}{6.00000000} \\
\hline & $\begin{array}{l}\text { Spin Coupling } \\
\text { Constant }\end{array}$ & \multicolumn{2}{|c|}{$\begin{array}{c}\text { Electronic Angular Momentum } \\
\text { Quantum Number }\end{array}$} & $\begin{array}{l}\text { Rotational } \\
\text { Constant, } D_{e} \\
\left(\mathrm{~cm}^{-1}\right)\end{array}$ & $\begin{array}{l}\text { Rotational } \\
\text { Constant, } \beta_{\mathrm{e}} \\
\quad\left(\mathrm{cm}^{-1}\right)\end{array}$ & $\begin{array}{l}\text { Dissociation } \\
\text { Energy, } \mathrm{D}_{\circ} \\
\left(\mathrm{cm}^{-1}\right)\end{array}$ & \multicolumn{2}{|c|}{$\begin{array}{l}\text { Internuclear Distance at Equilibrium } \\
\text { Position, } \mathrm{r}_{\mathrm{e}} \\
(\mathrm{cm})\end{array}$} \\
\hline Upper State & -16.90 & \multicolumn{2}{|c|}{1} & $6.74 \times 10^{-6}$ & $0.103 \times 10^{-6}$ & $2.95 \times 10^{4}$ & \multicolumn{2}{|c|}{$1.2661 \times 10^{-8}$} \\
\hline \multirow[t]{2}{*}{ Lower State } & -16.40 & \multicolumn{2}{|c|}{1} & $6.44 \times 10^{-6}$ & 0.0 & $4.89 \times 10^{4}$ & \multicolumn{2}{|c|}{$1.3119 \times 10^{-8}$} \\
\hline & $\begin{array}{l}\text { Electronic } \\
\text { Degeneracy }\end{array}$ & $\begin{array}{c}\text { Electronic } \\
\text { Term Energy } \\
\left(\mathrm{cm}^{-1}\right)\end{array}$ & $\begin{array}{l}\text { Vibrational } \\
\text { Spectroscopic } \\
\text { Constant, } \omega_{\mathrm{e}} \\
\left(\mathrm{cm}^{-1}\right)\end{array}$ & $\begin{array}{c}\text { Vibrational } \\
\text { Spectroscopic } \\
\text { Constant, } \omega_{\mathrm{e}} \mathrm{x}_{\mathrm{e}} \\
\left(\mathrm{cm}^{-1}\right)\end{array}$ & $\begin{array}{c}\text { Vibrational } \\
\text { Spectroscopic } \\
\text { Constant, } \omega_{\mathrm{e}} \mathrm{y}_{\mathrm{e}} \\
\left(\mathrm{cm}^{-1}\right)\end{array}$ & $\begin{array}{c}\text { Vibrational } \\
\text { Spectroscopic } \\
\text { Constant, } \omega_{\mathrm{e}} \mathrm{z}_{\mathrm{e}} \\
\left(\mathrm{cm}^{-1}\right)\end{array}$ & $\begin{array}{c}\text { Rotational } \\
\text { Constant, } \mathrm{B}_{\mathrm{e}} \\
\left(\mathrm{cm}^{-1}\right)\end{array}$ & $\begin{array}{c}\text { Rotational } \\
\text { Constant, } \alpha_{e} \\
\left(\mathrm{~cm}^{-1}\right)\end{array}$ \\
\hline Upper State & 6 & $2.00225 \times 10^{4}$ & 1788.22 & 16.440 & -0.5067 & 0.0 & 1.7527 & 0.01608 \\
\hline \multirow[t]{9}{*}{ Lower State } & 6 & 716.24 & 1641.35 & 11.67 & 0.0 & 0.0 & 1.63246 & 0.01661 \\
\hline & 1 & $5.50347 \times 10^{4}$ & 1671.50 & 40.02 & 0.248 & 0.0 & 1.7897 & 0.0387 \\
\hline & 1 & $4.323944 \times 10^{4}$ & 1829.57 & 13.94 & 0.0 & 0.0 & 1.8332 & 0.0196 \\
\hline & 6 & $4.079665 \times 10^{4}$ & 1106.56 & 39.260 & 2.8050 & -0.1271 & 1.1922 & 0.0242 \\
\hline & 2 & $3.42613 \times 10^{4}$ & 1809.1 & 15.81 & -4.02 & 0.0 & 1.7834 & 0.0180 \\
\hline & 3 & $1.33121 \times 10^{4}$ & 1961.6 & 13.7 & 0.0 & 0.0 & 1.87 & 0.0 \\
\hline & 2 & 8391.00 & 1608.35 & 12.078 & -0.01 & 0.0 & 1.61634 & 0.01686 \\
\hline & 3 & 6434.27 & 1470.45 & 11.19 & 0.028 & 0.0 & 1.49852 & 0.01634 \\
\hline & 1 & 0.0 & 1854.71 & 13.34 & -0.172 & 0.0 & 1.81984 & 0.01765 \\
\hline
\end{tabular}




\section{Computational Results}

- RP-1 Combustion at O/F Ratio of 2.0

- $3000 \mathrm{~K}$

- $1 \mathrm{~atm}$

- $10 \mathrm{~cm}$ Pathlength

- 300 to $430 \mathrm{~nm}$ for Atomic Emission 
Table 2. Assumed Free Atomic Number Density for Atomic Species

\begin{tabular}{|c|c|}
\hline Element & $\begin{array}{c}\text { Free Atomic Number } \\
\text { Density } \\
\text { (in free atoms/cm }\end{array}$ \\
\hline $\mathrm{Ni}$ & $5.0 \times 10^{12}$ \\
\hline $\mathrm{Fe}$ & $1.0 \times 10^{12}$ \\
\hline $\mathrm{Cr}$ & $2.0 \times 10^{11}$ \\
\hline $\mathrm{Co}$ & $1.0 \times 10^{13}$ \\
\hline $\mathrm{Mn}$ & $2.0 \times 10^{11}$ \\
\hline $\mathrm{Cu}$ & $1.0 \times 10^{12}$ \\
\hline $\mathrm{Ag}$ & $1.0 \times 10^{12}$ \\
\hline $\mathrm{Pd}$ & $1.0 \times 10^{12}$ \\
\hline $\mathrm{Al}$ & $1.0 \times 10^{12}$ \\
\hline $\mathrm{Ca}$ & $1.0 \times 10^{10}$ \\
\hline
\end{tabular}




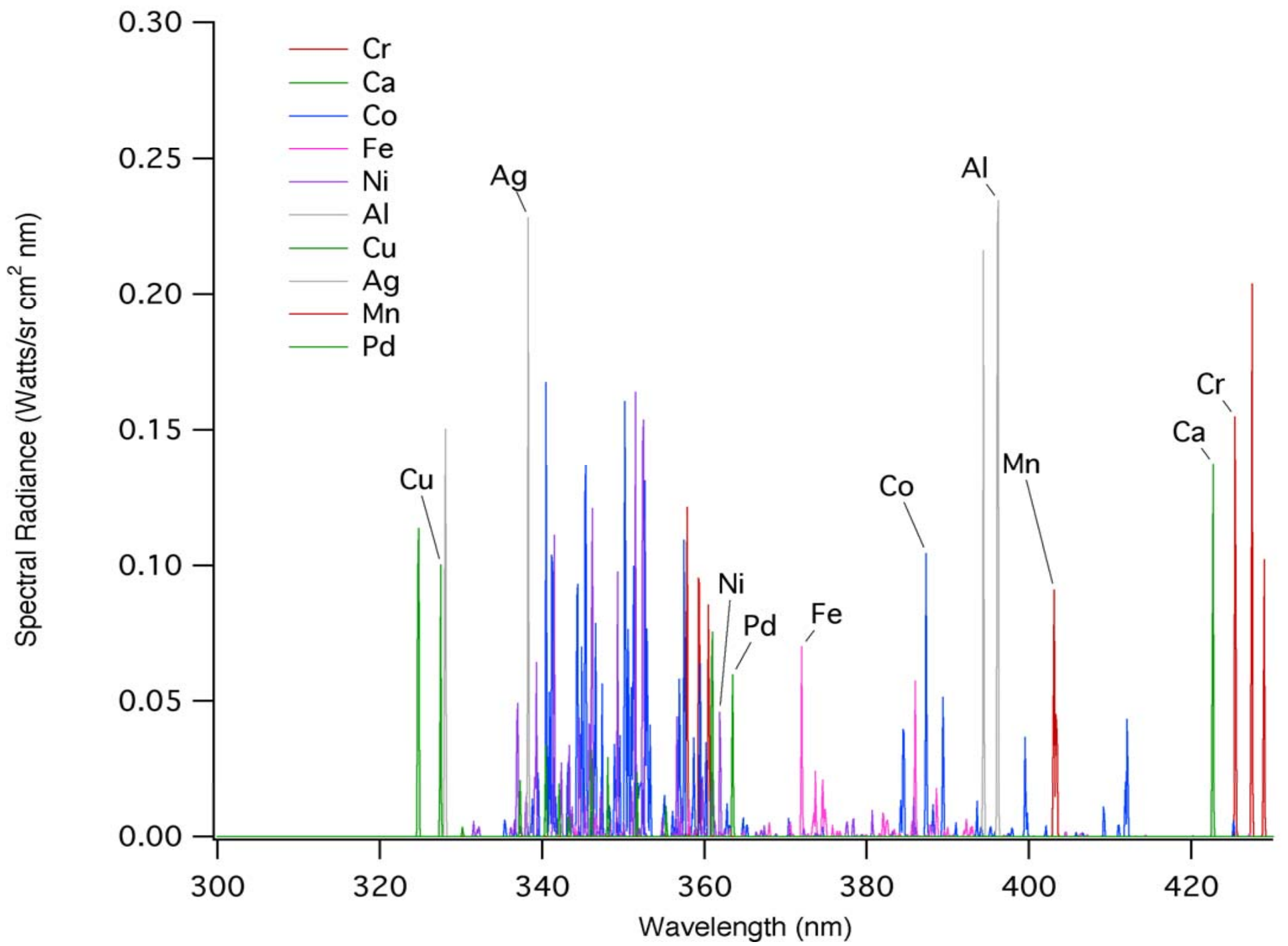

Figure 1. Computed Spectrum for Ni, Fe, Cr, Co, Al, Mn, Cu, Ca, Ag, and Pd at $3000 \mathrm{~K}$ for $10 \mathrm{~cm}$ Pathlength, 300.0 to $430.0 \mathrm{~nm}$. Tagged Lines for All Ten Elements Correspond to the SSME EDC. 


\section{$\mathrm{C}_{2}$ Swan Band System}

- Present in All Hydrocarbon Combustion

- Bands Included: $(0,0),(0,1),(0,2),(1,0),(1,1),(1,2),(1,3),(2,0)$, $(2,1),(2,2),(2,3),(2,4),(3,1),(3,2),(3,3),(3,4),(3,5),(4,2),(4,3)$, $(4,4),(4,5),(5,3),(5,4),(6,4),(6,5),(7,5),(7,6),(8,6)$, and $(8,7)$

- Spectral Emission Range: 422.9 to $619.7 \mathrm{~nm}$

- Affected Elements/Lines: $\operatorname{Cr}(425.43,427.48$, and $428.97 \mathrm{~nm})$ 


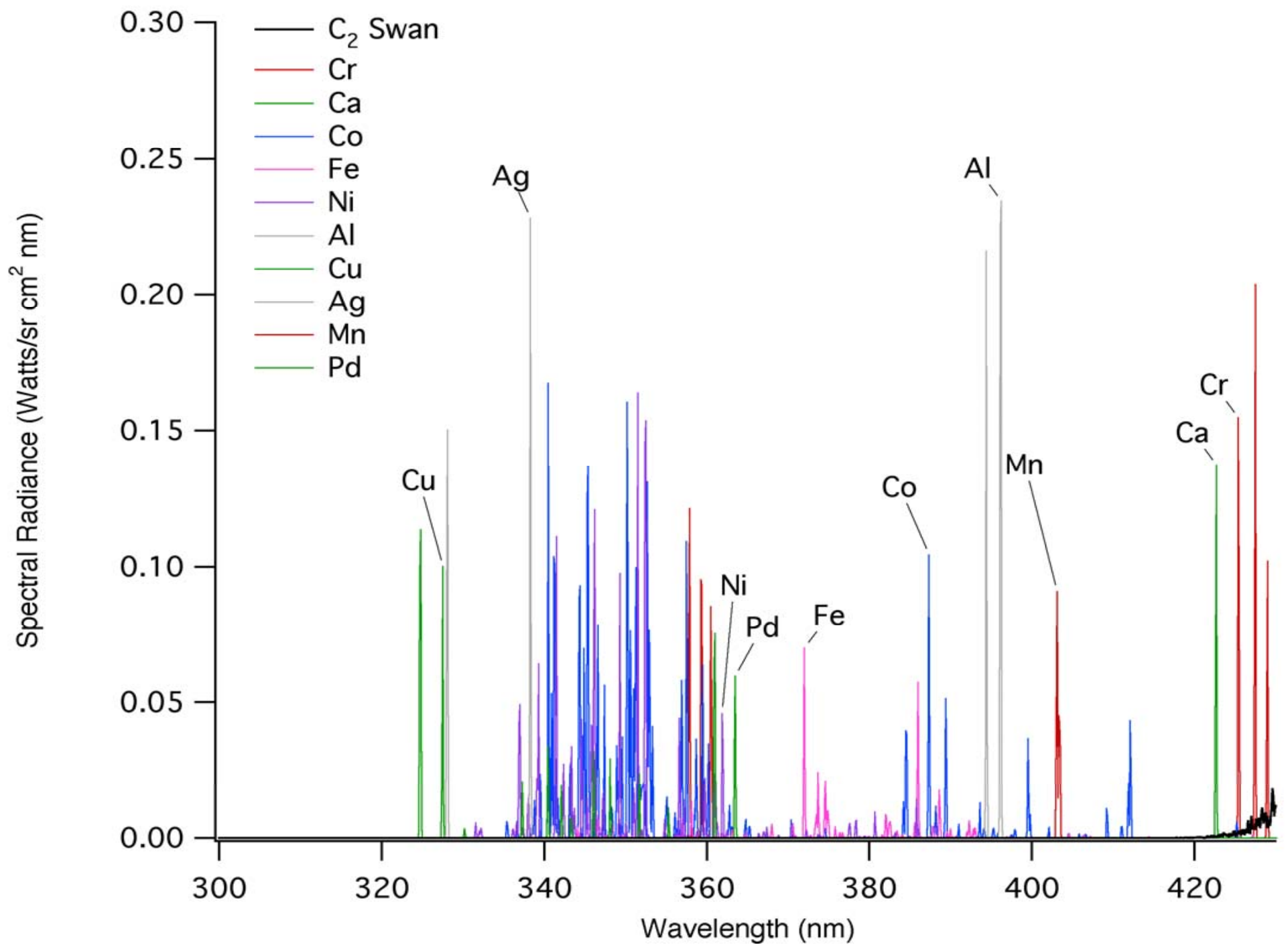

Figure 2. Computed Spectrum for $\mathrm{Ni}, \mathrm{Fe}, \mathrm{Cr}, \mathrm{Co}, \mathrm{Al}, \mathrm{Mn}, \mathrm{Cu}, \mathrm{Ca}, \mathrm{Ag}, \mathrm{Pd}$ and $\mathrm{C}_{2}$ Swan Band System at $3000 \mathrm{~K}$ for $10 \mathrm{~cm}$ Pathlength, 300.0 to $430.0 \mathrm{~nm}$. $C_{2}$ Concentration in the Plume is $1 \times 10^{14}$ Molecules $/ \mathrm{cm}^{3}$. 


\section{CH $3900 \AA$ Band System}

- Common Intermediate Radical

- Bands Included: $(0,0),(0,1),(0,2),(1,0),(1,1),(1,2),(1,3),(2,0)$, $(2,1),(2,2),(2,3),(3,0),(3,1),(3,2),(3,3),(4,1),(4,2)$, and $(4,3)$

- Spectral Emission Range: 346.1 to $540.5 \mathrm{~nm}$

- Strongest Emission:

386.6 to $413.4 \mathrm{~nm}$

- Affected Elements/Lines: Al (394.40 and $396.15 \mathrm{~nm})$

$\mathrm{Mn}(403.08,403.31$, and $403.45 \mathrm{~nm})$

$\mathrm{Ca}(422.67 \mathrm{~nm})$

Co $(387.31 \mathrm{~nm})$ 


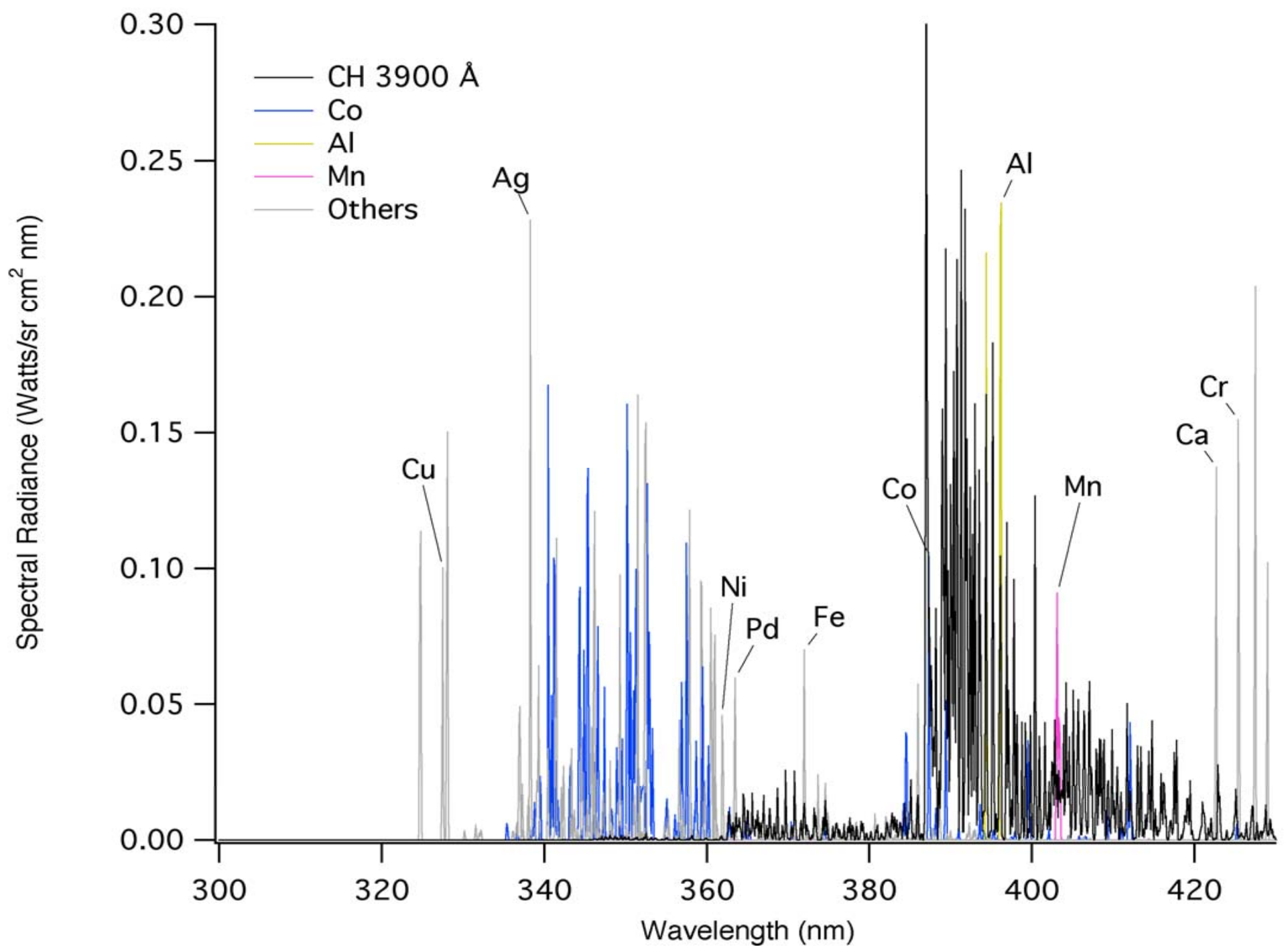

Figure 3. Computed Spectrum for $\mathrm{Ni}, \mathrm{Fe}, \mathrm{Cr}, \mathrm{Co}, \mathrm{Al}, \mathrm{Mn}, \mathrm{Cu}, \mathrm{Ca}, \mathrm{Ag}, \mathrm{Pd}$, and $\mathrm{CH} 3900 \AA$ Band System at $3000 \mathrm{~K}$ for $10 \mathrm{~cm}$ Pathlength, 300.0 to $430.0 \mathrm{~nm}$. CH Concentration in the Plume is $5 \times 10^{14}$ Molecules $/ \mathrm{cm}^{3}$. 


\section{$\mathrm{CH} 4300 \AA$ Band System}

- Bands Included: $(0,0),(0,1),(0,2),(1,0),(1,1),(1,2),(1,3)$, $(2,0),(2,1),(2,2),(2,3),(2,4),(3,1),(3,2),(3,3),(4,2),(4,4),(4,5)$, $(5,3),(5,4)$, and $(5,5)$

- Spectral Emission Range: 409.2 to $453.6 \mathrm{~nm}$ at $3000 \mathrm{~K}$

- Strongest Emission:

420 to $440 \mathrm{~nm}$

- Affected Elements/Lines: $\operatorname{Cr}(425.43,427.48$, and $428.97 \mathrm{~nm})$ 


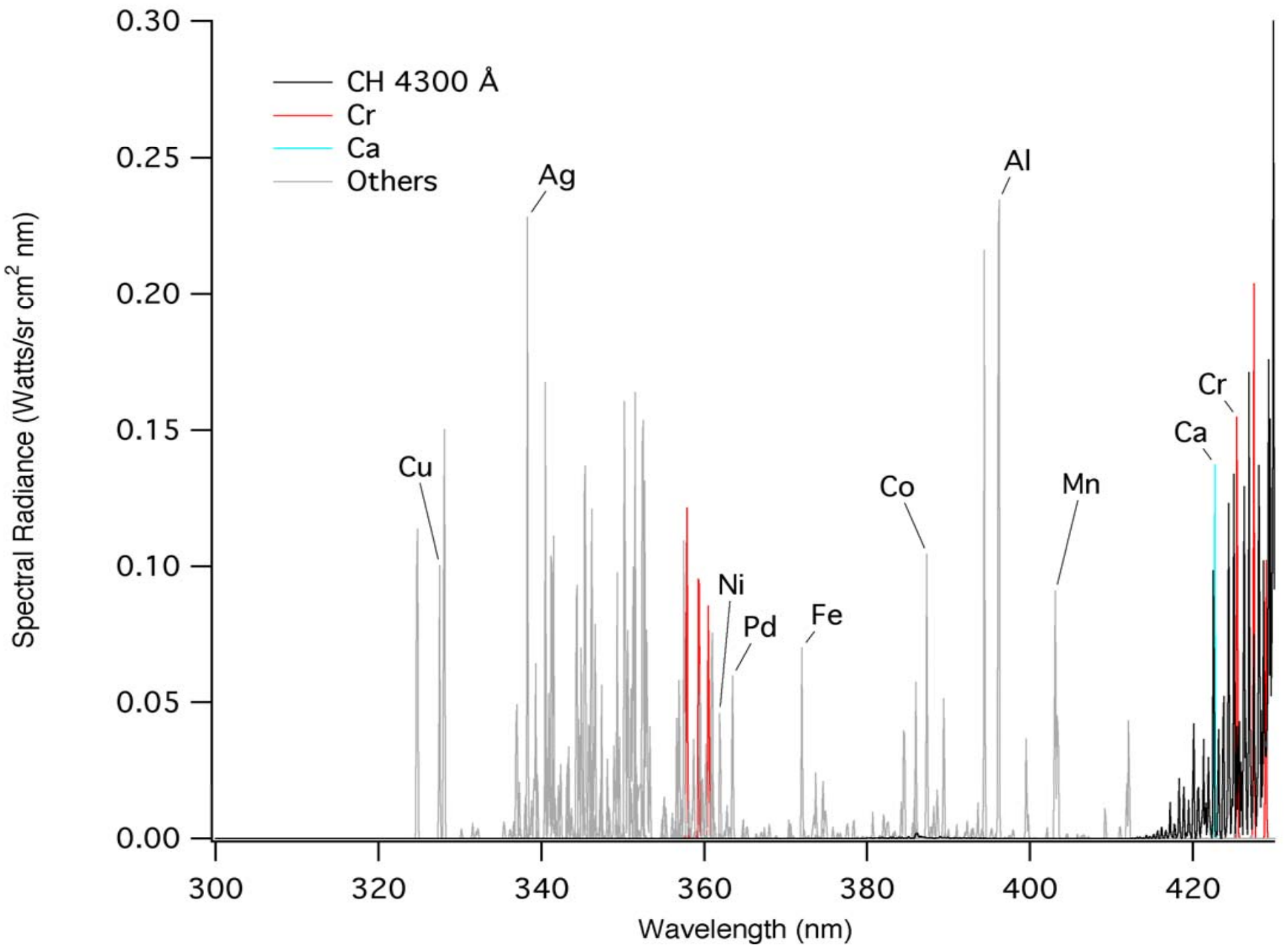

Figure 4. Computed Spectrum for $\mathrm{Ni}, \mathrm{Fe}, \mathrm{Cr}, \mathrm{Co}, \mathrm{Al}, \mathrm{Mn}, \mathrm{Cu}, \mathrm{Ca}, \mathrm{Ag}, \mathrm{Pd}$, and $\mathrm{CH} 4300 \mathrm{~A}$ Band System at $3000 \mathrm{~K}$ for $10 \mathrm{~cm}$ Pathlength, 300.0 to $430.0 \mathrm{~nm}$. CH Concentration in the Plume is $1 \times 10^{14} \mathrm{Molecules} / \mathrm{cm}^{3}$. 


\section{CN Violet Band System}

- Free Radical in Mixing Layer

- Bands Included: $(0,0),(0,1),(0,2),(0,3),(1,0),(1,1),(1,3),(2,1)$, $(2,2),(2,3),(2,4),(3,1),(3,2),(3,3),(3,4),(3,5),(4,3),(4,4),(4,5)$, $(4,6),(5,4),(5,5)$, and $(5,6)$

- Spectral Emission Range: 350.9 to $358.7 \mathrm{~nm}$ 373.9 to $388.0 \mathrm{~nm}$ 406.1 to $421.1 \mathrm{~nm}$

- Strongest Emission:

380 to $388 \mathrm{~nm}$

- Affect Elements/Lines:

Co $(387.31 \mathrm{~nm})$ 


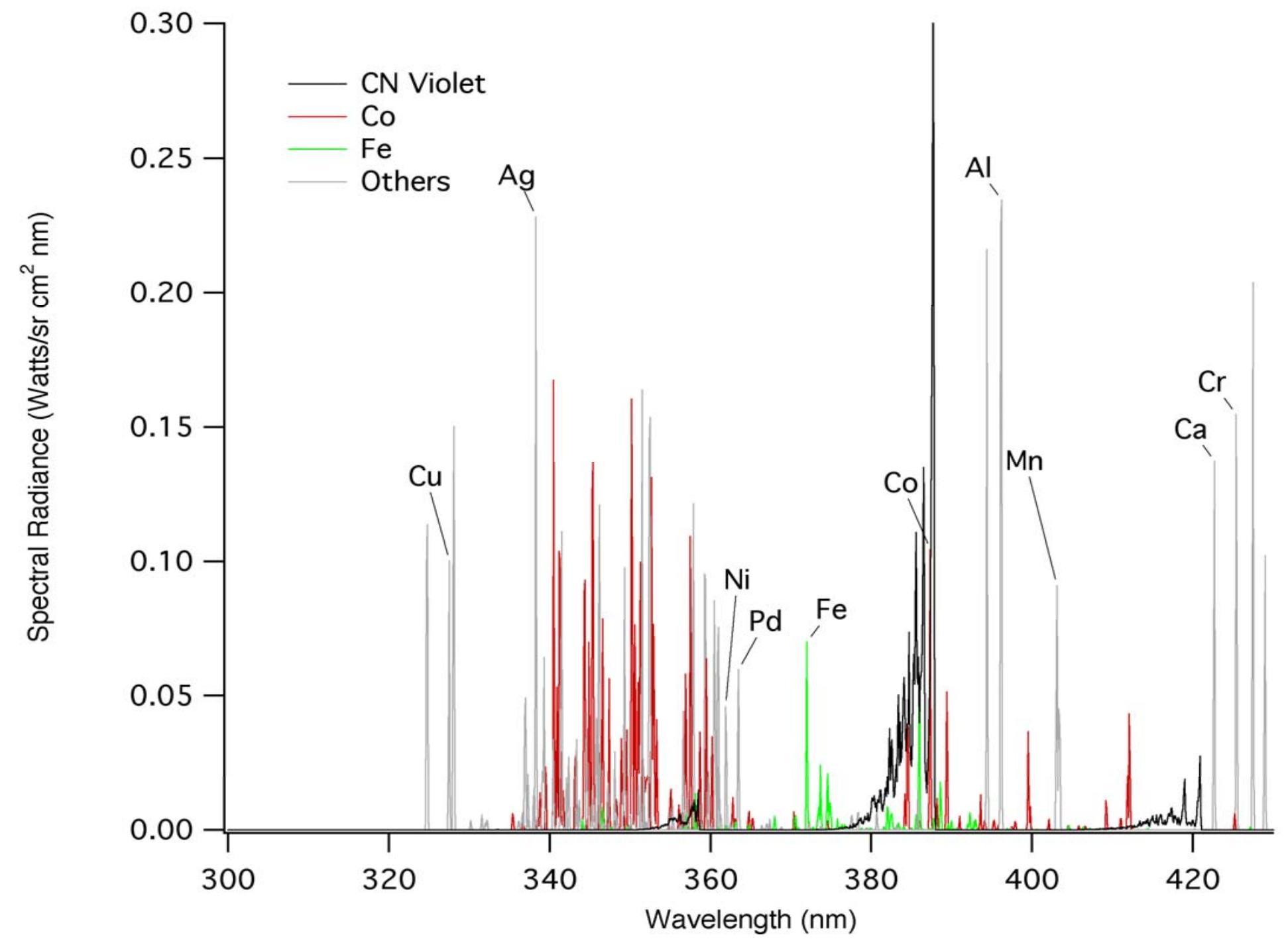

Figure 5. Computed Spectrum for $\mathrm{Ni}$, Fe, $\mathrm{Cr}, \mathrm{Co}, \mathrm{Al}, \mathrm{Mn}, \mathrm{Cu}, \mathrm{Ca}, \mathrm{Ag}, \mathrm{Pd}$, and $\mathrm{CN}$ Violet Band System at $3000 \mathrm{~K}$ for $10 \mathrm{~cm}$ Pathlength, 300.0 to $430.0 \mathrm{~nm}$. CN Concentration in the Plume is $2 \times 10^{13}$ Molecules $/ \mathrm{cm}^{3}$. 


\section{CO+ Comet-Tail Band System}

- Product of Incomplete Combustion

- Bands Included: $(0,0),(0,1),(0,2),(0,3),(0,4),(0,5),(1,0),(1,1)$, $(1,2),(1,3),(1,4),(1,5),(2,0),(2,1),(2,2),(3,0),(3,1),(3,2),(3,3)$, $(4,0),(4,1),(4,2),(5,0),(5,1),(5,2),(6,0),(6,1)$, and $(7,1)$

- Spectral Emission Range: 341.2 to $802.4 \mathrm{~nm}$

- Strongest Emission: $\quad 400$ to $430 \mathrm{~nm}$

- Affected Elements/Lines: $\quad \mathrm{Mn}(403.08,403.31$, and $403.45 \mathrm{~nm})$

Ca $(422.67 \mathrm{~nm})$

Cr $(425.43,427.48$, and $428.97 \mathrm{~nm})$ 


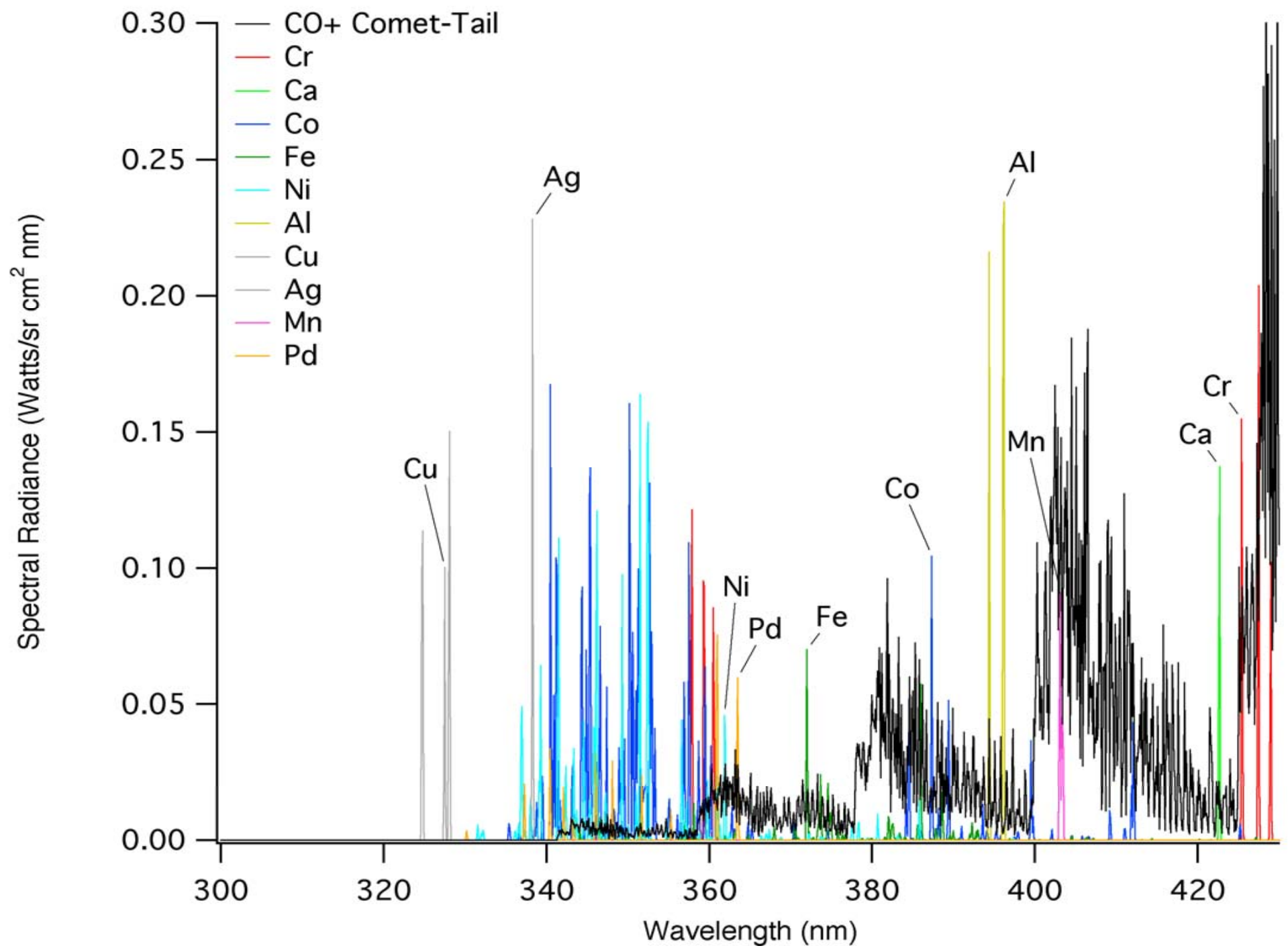

Figure 6. Computed Spectrum for $\mathrm{Ni}, \mathrm{Fe}, \mathrm{Cr}, \mathrm{Co}, \mathrm{Al}, \mathrm{Mn}, \mathrm{Cu}, \mathrm{Ca}, \mathrm{Ag}, \mathrm{Pd}$, and CO+Comet-Tail Band System at $3000 \mathrm{~K}$ for $10 \mathrm{~cm}$ Pathlength, 300.0 to $430.0 \mathrm{~nm}$. CO+ Concentration in the Plume is $4 \times 10^{15}$ Molecules $/ \mathrm{cm}^{3}$. 
"Best" Atomic Lines for the Engine Health Monitoring

\begin{tabular}{|c|c|}
\hline Element & Wavelength (nm) \\
\hline $\mathrm{Cu}$ & 327.40 \\
\hline $\mathrm{Ag}$ & 338.29 \\
\hline $\mathrm{Co}$ & 350.23 \\
\hline $\mathrm{Ni}$ & 361.94 \\
\hline $\mathrm{Pd}$ & 363.47 \\
\hline $\mathrm{Fe}$ & 371.99 \\
\hline $\mathrm{Al}$ & 396.15 \\
\hline $\mathrm{Mn}$ & 403.08 \\
\hline $\mathrm{Ca}$ & 422.67 \\
\hline $\mathrm{Cr}$ & 425.43 \\
\hline
\end{tabular}




\section{Methane Thruster Test Program (MTTP) Features}

- $50 \mathrm{lb}$ Thrust Gaseous Oxygen/Gaseous Methane Thruster

- MTTP Operational since March 2006

- Spectral Instrumentation: High-Resolution OMA System, 310-436 nm Broadband Spectrometer, 300-825 nm

- Optical System Details in the Paper

- O/F Ratio of 2.0 and 2.5 for Fuel-Rich Combustion 


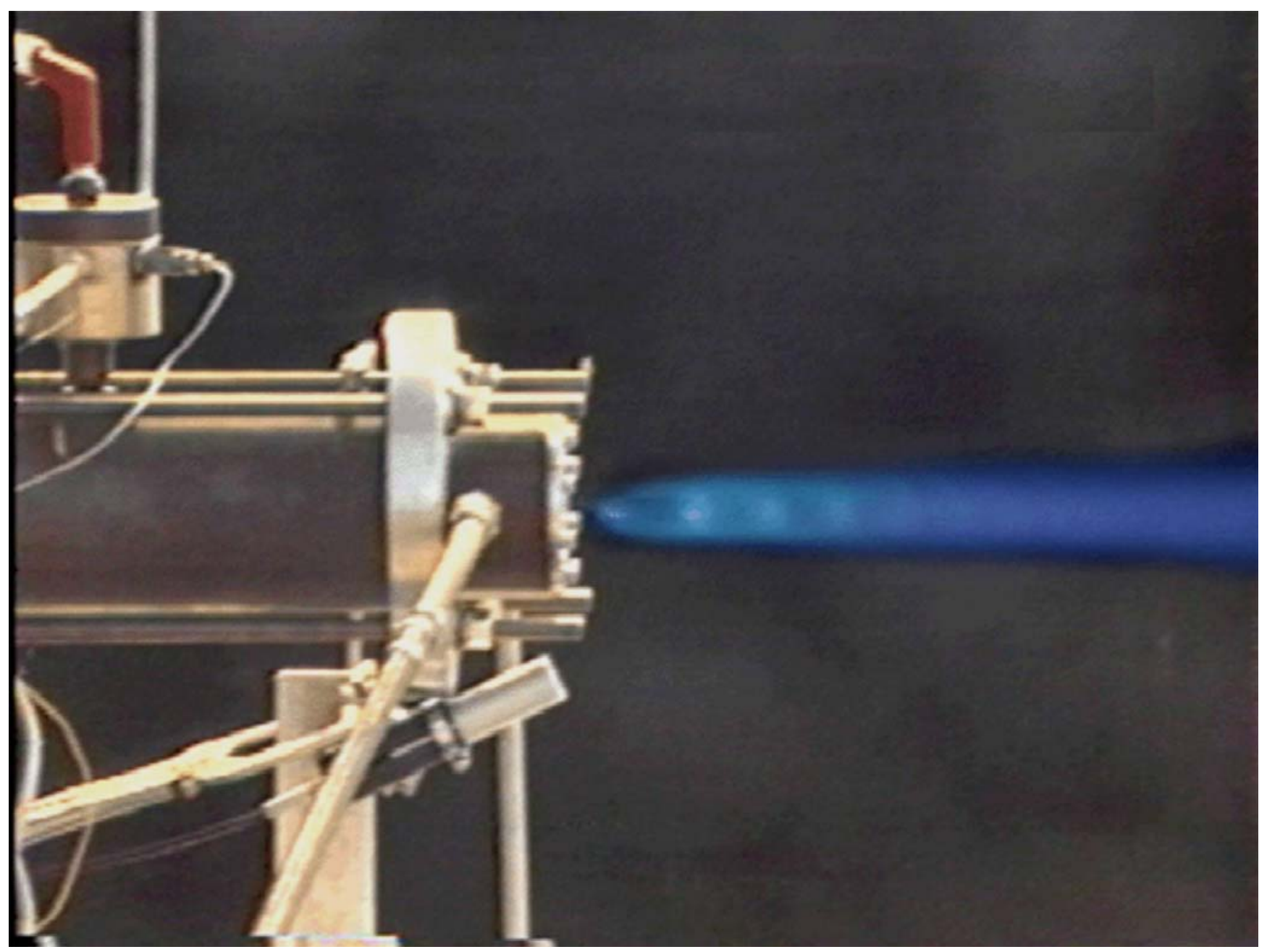

Figure 7. $50 \mathrm{lb}$ Thrust Gaseous Oxygen/Gaseous Methane Thruster 


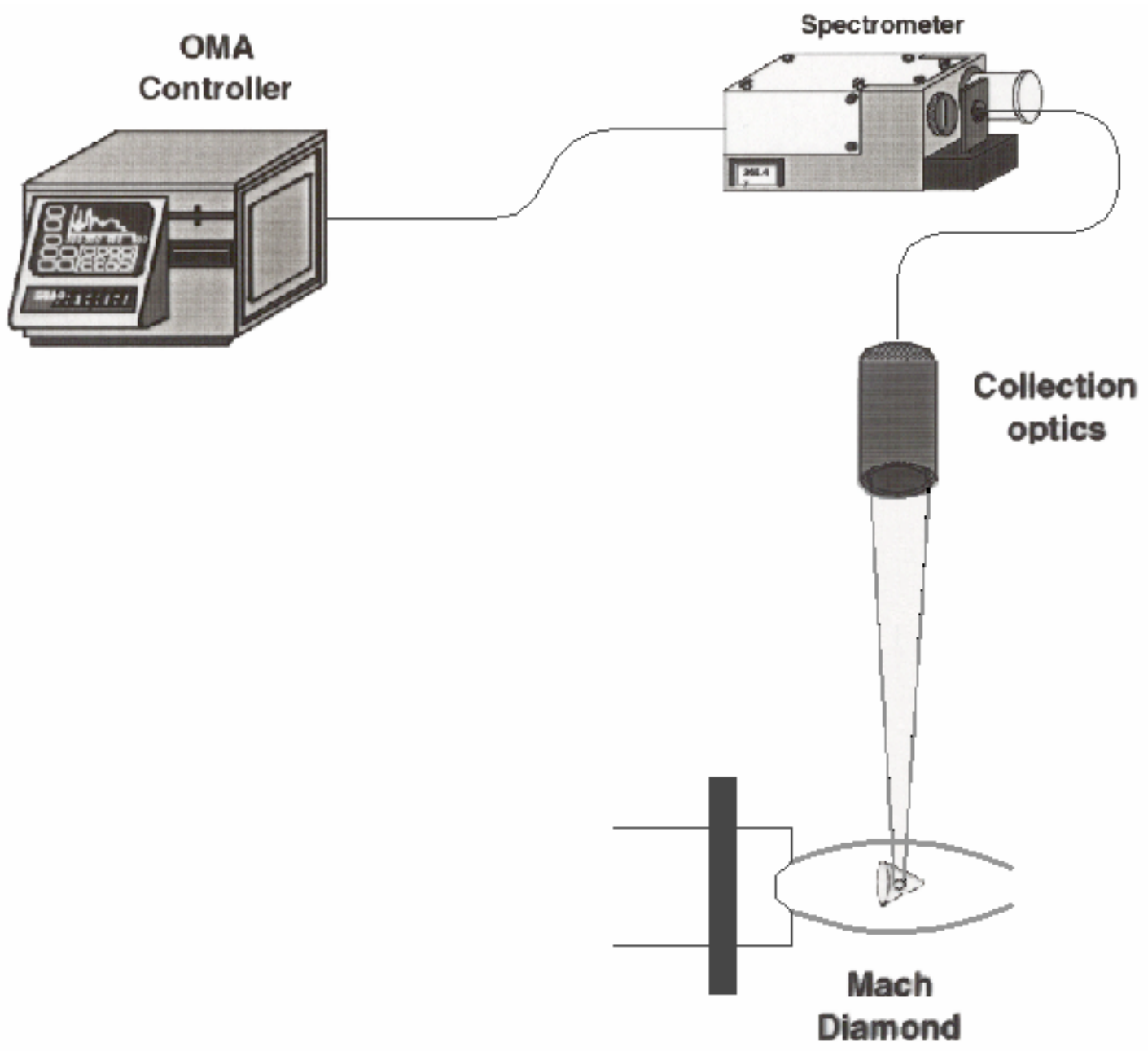

Figure 8. Atomic Emission Spectroscopic System Configuration 


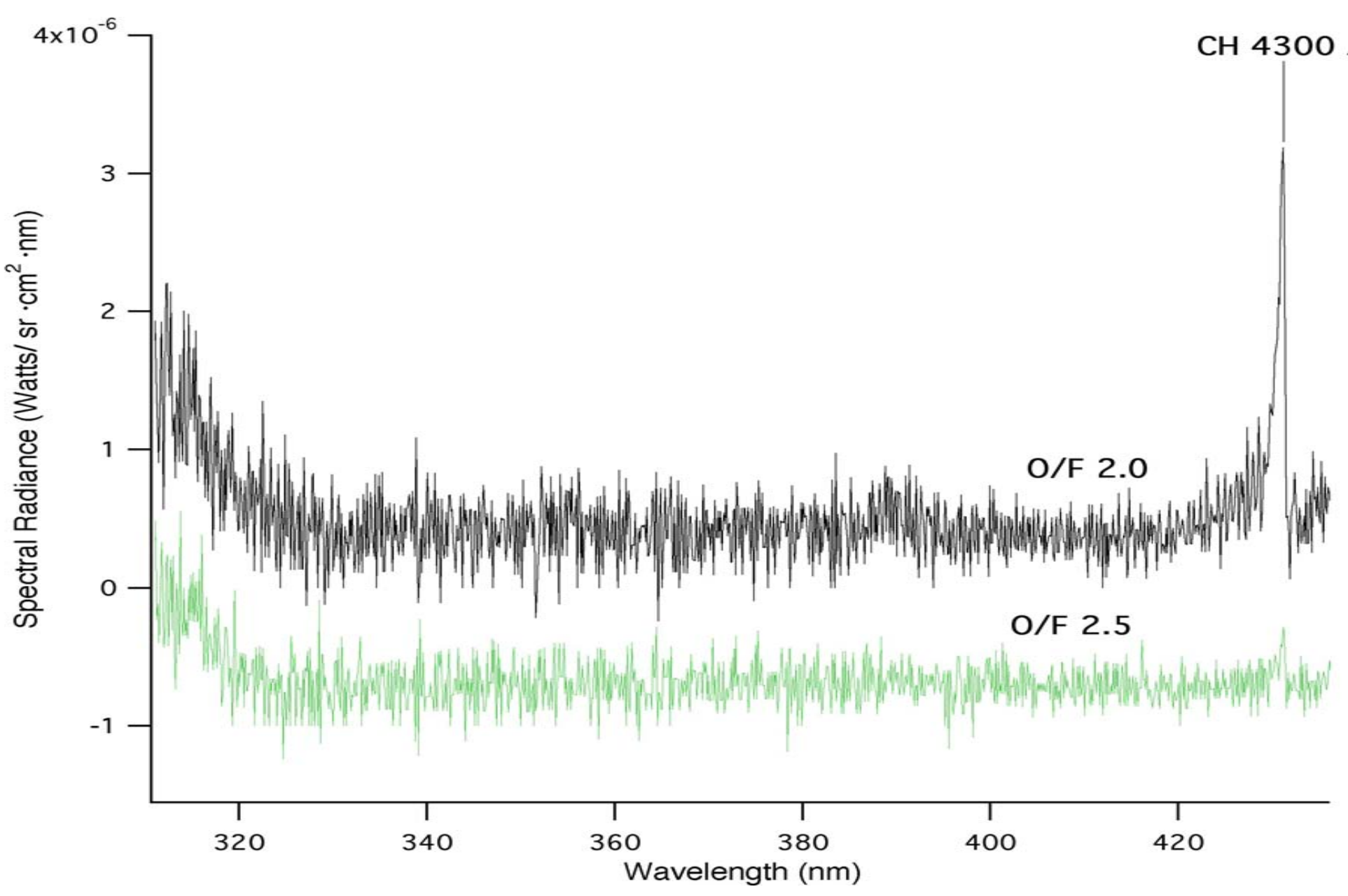

Figure 9. High Resolution Spectra from Tests A and B showing CH 4300 A Band System Emissions at O/F Ratios of 2.0 and 2.5, Respectively. Note the Weaker Signature of CH 3900 A Band System Discernable for Test A in O/F 2.0 Spectra. 


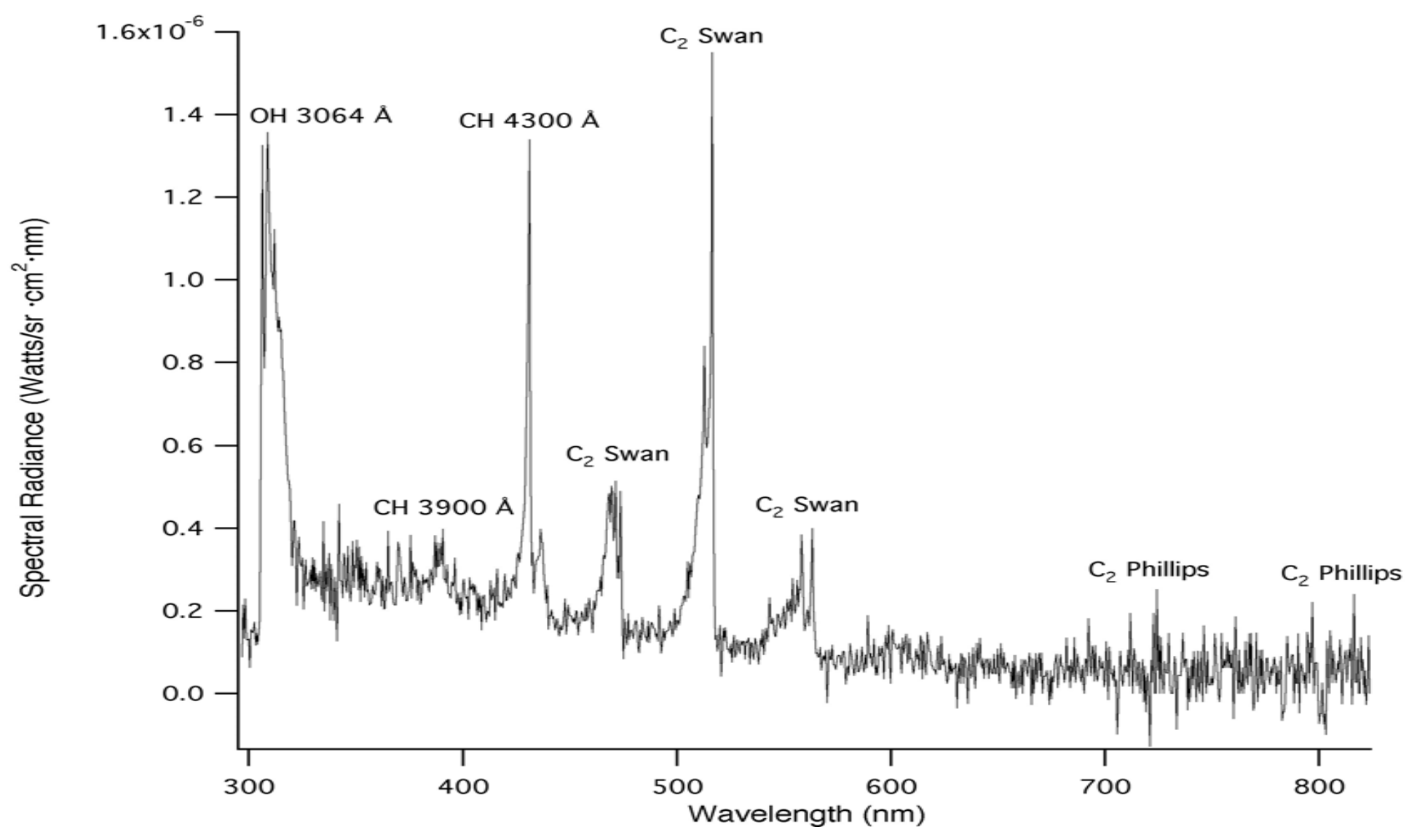

Figure 10. Broadband Spectra from Test A Displaying the Emission Band Systems of OH 3064 A, $\mathrm{CH} 3900 \mathrm{~A}, \mathrm{CH} 4300 \AA$ A, $\mathrm{C}_{2}$ Swan and $\mathrm{C}_{2}$ Phillips. 


\section{Summary and Recommendations}

1. RPSSC is Complete for Hydrocarbon Fuels

2. Tentative "Best" Lines for the Engine Health Monitoring for $\mathrm{Fe}, \mathrm{Ni}, \mathrm{Cr}, \mathrm{Co}, \mathrm{Mn}, \mathrm{Ag}, \mathrm{Cu}, \mathrm{Pd}, \mathrm{Al}$, and $\mathrm{Ca}$

3. MTTP is Operational

4. Future Work

a. Detection Sensitivity Limits

b. Broadband Spectral Region with all 11 Electronic Bands for $Y$ and Ti Monitoring

c. MTTP Measurements with Metallic Dopants in the Combustion Chamber

d. Free Atomic Factor for the 10 Elements in the $\mathrm{CH}_{4}$ and RP-1 Engine Exhaust Plume 